\title{
Strategies of polyhydroxyalkanoates modification for the medical application in neural regeneration/nerve tissue engineering
}

\author{
Xiaoyun Lu ${ }^{1}$, Leilei Wang ${ }^{1}$, Zhiqian Yang ${ }^{1,2}$, Haixia Lu $^{2 *}$ \\ ${ }^{1}$ Key Laboratory of Biomedical Information Engineering of Ministry of Education, School of Life Science and Technology, Xi'an \\ Jiaotong University, Xi'an, China \\ ${ }^{2}$ Institute of Neurobiology, Xi'an Jiaotong University College of Medicine, Xi'an, China \\ Email:
}

Received 17 April 2013; revised 23 May 2013; accepted 30 May 2013

Copyright (C) 2013 Xiaoyun Lu et al. This is an open access article distributed under the Creative Commons Attribution License, which permits unrestricted use, distribution, and reproduction in any medium, provided the original work is properly cited.

\begin{abstract}
Neural regeneration was once considered to be impossible, especially in the central nervous system where neural regeneration comprise the generation of new neurons, glia, axons, myelin, and synapses. Until recently, neural stem cells/neural progenitor cells (NSCs/NPCs) were identified from various areas of brain and brought hopes to the neural repair and regeneration. Tissue engineering has revolutionized the current neural regeneration technology and it has become a pioneering interdisciplinary field in the areas of biomedical research. Polyhydoxyalkanoate (PHA) as one of biodegradable material has been successfully used as tissue engineering materials. It has also been applied in nerve tissue engineering due to the high biocompatibility and low cytotoxicity. Over the past 10 years, different kinds of modification strategies have been undertaken to improve the properties of PHA to fit the requirements from various fields. Several members of PHA family have been attempted for neural regeneration. This article reviewed the recent modification strategies for improving the properties of PHA and highlighted the pioneer applications in neural regeneration.
\end{abstract}

Keywords: PHA; Modification; Neural Regeneration; Nerve Tissue Engineering

\section{INTRODUCTION}

Neural regeneration is a very complex bioprocess, and it was considered to be impossible. Neural regeneration differs between the peripheral nervous system (PNS) and the central nervous system (CNS) by the functional

${ }^{*}$ Corresponding author. mechanisms and especially the extent and speed. The lack of regenerative properties of the mammalian CNS, especially in the spinal cord, may be attributable to a combination of factors, including the inhibitory character of CNS myelin and injury-induced glial scars, the apparent inability of endogenous adult cells to induce de novo neurogenesis upon injury [1] and the lack of sufficient trophic support [2]. Although introducing an appropriate environment into the injured site via applying neurotrophic factors and fetal material transplantation could be effective, shortage of donors and growing ethical problems precluded the practical clinical application of this approach [3-5]. Until recently, as a result of remarkable advances in neuroscience, NSCs and NPCs were identified and used as a feasible transplant material. NSCs/ NPCs are self-renewable and multipotent cells that could give rise to both neuron and glia [6]. Therefore, NSCs/ NPCs transplantation has been considered as a high promising strategy to improve neural regeneration [7-11]. However, inadequate number of NSCs survived in situ of injury has embarrassed the application of NSCs therapy in a certain level [8-10].

A newly emerging field, tissue engineering has been considered as a means to facilitate neural repair. Various kinds of biomaterials have been explored to act as supporting materials in nerve tissue engineering, and shown their superiorities [11-13]. PHAs have been demonstrated to be a family of biodegradable polyesters with good biodegradability and noncytotoxicity, and therefore they have been developed as bio-implantable materials for many medical applications [14]. However, some obstacles, such as the surface hydrophobicity, have hindered the extensive usage of PHA in medical applications. During the past 10 years, many efforts have been made to modify the surface of PHA in order to improve biocompatibility, enhance cell adhesion and extracellular 
matrix protein distribution. Those physical, chemical and biological strategies towards PHA modification could also be used to develop suitable PHA scaffold for repairing neural injury which will be discussed in the following sections.

\section{PHA FAMILY MEMBERS FOR TISSUE ENGINEER APPLICATION}

PHAs are a family of biodegradable polyesters synthesized by bacteria as intracellular carbon and energystorage compounds. More than 150 kinds of PHAs consisting of various co-monomers have been reported [15]. However, only few of them have been considered for commercial production, such as poly (3-hydroxybutyrate) (PHB), poly (3-hydroxybutyrate-co-3-hydroxyhexanoate) (PHBHHx) and poly (3-hydroxybutyrate-co-3-hydroxy-valerate) (PHBV). PHB is the most thoroughly investigated member of the PHA family and it has been demonstrated to have good biocompatibility to adrenocortical cells [16], osteoblasts, epithelial cells [17], fibroblasts, endothelium cells and isolated hepatocytes [18]. However, the high brittleness and low degradation have limited its application. The copolymer of 3-hydroxybutyrate (3-HB) and 3-hydroxyvalerate (3-HV), PHBV is another family member of PHAs. PHBV fibers promoted re-epithelialization [19]. Combined with some chemical cues, the microtopographies of PHBV changed and resulted in improvement of osseointegration [20]. PHBHHx is another family member of PHA which is considered as a very promising tissue engineering material with adjustable mechanical properties, biocompatibility and biodegradability [14]. In comparison with PHB and PHBV, PHBHHx has superior physical and mechanical properties. The lower melting point and the presence of middle-chain-length monomer, 3-hydroxyhexanoate (3-HHx) fraction insure it to have a higher flexibility and better impact strength [21]. In addition, PHBHHx has better biocompatibility with smooth muscle cells [22], fibroblasts [23], rabbit articular cartilage-derived chondrocytes [24], osteoblasts [25], bone marrow cells [26], and neural stem cells [27]. Poly (3-hydroxybutyrate-co-3-hydroxyvalerate-co-3-hydroxyhexanoate) (PHBVHHx) is a new member of PHA family which consisting of 3-HB, $3-\mathrm{HV}$ and 3-HHx. PHBVHHx (containing $5.3 \mathrm{~mol} \% \mathrm{HV}$ and $10.2 \mathrm{~mol} \% \mathrm{HHx}$ ) can be cast into a highly flexible film [28] due to its improved mechanical properties over PHB and PHBHHx. In addition, it has a rougher surface and a higher hydrophobicity than PHBHHx. There is a published data showed that human bone marrow mesenchymal stem cells (MSCs) adhered better on PHBVHHx (containing $3.9 \mathrm{~mol} \% \mathrm{HV}$ and $13.4 \mathrm{~mol} \% \mathrm{HHx}$ ) films than PHBHHx films, and undertook osteogenic differentiation preferably [29]. More details about the application of polyhydroxyalkanoates as tissue engineering materials have been reviewed by Chen [14].

Nevertheless, although PHAs have been demonstrated to be biocompatible, biodegradable and nontoxic, and have been applied for tissue engineering, there were still some properties needed to be elevated before apply them in neural regeneration. For example, poor hydrophilic property of PHA [30,31] dramatically reduced the cell attachment and resulted in the less efficiency of cell replacement. Since PHAs were native polymers whose thermal and mechanical properties could be adjusted from thermoplastic to elastomeric depending on various compositions of their monomeric components. Many approaches including blending with other materials, and physico-chemical and biological modification have been undertaken to adjust the mechanical characters and/or biocompatibility of PHA for the purpose of better biocompatibility.

\section{APPROACHES HAVE BEEN UNDERTAKEN TO IMPROVE THE HYDROPHILIC PROPERTIES OF PHA}

In general, hydrophilicity strongly influences cell attachment to a material. The higher the hydrophilicity of a material surface, the stronger the cells attached [30]. As a hydrophobic polymer material, poor hydrophilic property of PHA hinders its extensive usage in medical application. Therefore, it is necessary to improve the hydrophilic property of PHA to meet rigorous tissue engineering requirement. Some of the useful approaches in improving hydrophilic properties of PHB and PHBHHx were listed as below (Table 1).

Surface treatment is a possible and effective way to improve the hydrophilic property of PHAs. Different strategies, including grafting technique [32], ultraviolet treatment [33], plasma treatment [34] and surface hydrolysis [35], have been applied to improve the hydrophilicity of polymer materials. However, these techniques still have some disadvantages. For example, grafting technique make the material surface very unstable because of the very weak interaction between bulk polymer and the bioactive molecules [32]. The direct ultraviolet radiation significantly reduces the mechanical properties of polymer films and makes them very brittle [33]. Even the treatment with plasma could only increase the hydrophilicity of PHAs surface temporary and the surface tends to revert to the untreated state shortly after the treatment [34]. Alkali and lipase treatment have been demonstrated to be very effective to improve the wetting and adhesion property of polymer surface. $\mathrm{NaOH}$, acts as a hydrolysis catalyst and lipase is an enzyme specifically acting on ester bonds of polyesters. Shen et al. have reported that $\mathrm{NaOH}$ treatment improved the surface hydrophilic property of PHA and enhanced its blood compatibility [31]. It also has been demonstrated that $\mathrm{NaOH}$ 
Table 1. Modification strategies attempting to improve the hydrophilic properties of PHA.

\begin{tabular}{|c|c|c|c|}
\hline Material & Modification method & Improved properties (effect) & Ref. \\
\hline \multirow[t]{4}{*}{ PHB } & treated with lipase & transferred coralloid film surface into pore free (improved L929 cells growth) & {$[30,41]$} \\
\hline & coated with gelatin & (promoted adrenocortical cell grow and survive) & {$[37]$} \\
\hline & blending with PHBHHx & $\begin{array}{l}\text { decreased crystallinity, exposed more oxidized carbon atom on the surface (growth of } \\
\text { chondrocytes changed from spherical to flat) }\end{array}$ & {$[42]$} \\
\hline & coated with PhaP-RGD & $\begin{array}{l}\text { e improved hydrophilicity, enhanced attachment of fibroblasts (improved L929 and } \\
\text { NIH/3T3 cell growth) }\end{array}$ & {$[40]$} \\
\hline \multirow[t]{8}{*}{ PHBHHx } & $1 \mathrm{M} \mathrm{NaOH}$ & $\begin{array}{l}\text { increased roughness, surface free energyand the polarity of the film (promoted } \\
\text { MC3T3-E1 cells attachment and proliferation) }\end{array}$ & [43] \\
\hline & $\begin{array}{l}\text { lipase treatment hyaluronic } \\
\text { acid (HA) coating }\end{array}$ & $\begin{array}{l}\text { increased hydrophilic and smoothness (lipase treatment increased L929 cells growth } \\
\text { and HA coating reduced L929 cell attachment and proliferation) }\end{array}$ & [44] \\
\hline & UV radiation & $\begin{array}{l}\text { improved hydrophilic, but increased the brittleness and degradation of material } \\
\text { (increased L929 cells growth) }\end{array}$ & [33] \\
\hline & blending with gelatin & $\begin{array}{l}\text { increased the surface porosity and tensile strength, decreased the crystallinity (benifical } \\
\text { for cell growth) }\end{array}$ & [37] \\
\hline & $\begin{array}{l}\text { plasma treated and coated with } \\
\text { fibronectin }\end{array}$ & enhanced surface energy and hydrophilic (promoted HUVECs and SMCs growth) & [45] \\
\hline & grafted by maleic anhydride & $\begin{array}{l}\text { improved hydrophilicity, more thermo-stable and formed whorl-like structure on the } \\
\text { surface (increased L929 cells and HMECs attachment and proliferation) }\end{array}$ & [46] \\
\hline & PhaP-RGD modification & $\begin{array}{l}\text { improved hydrophilicity, enhanced attachment of fibroblasts (improved L929 and } \\
\text { NIH/3T3 cell growth) }\end{array}$ & [40] \\
\hline & PhaR-KQAGDV modification & $\begin{array}{l}\text { improved hydrophilicity, enhanced adhesion and proliferation of human vascular } \\
\text { smooth muscle cells }\end{array}$ & [38] \\
\hline
\end{tabular}

treatment could enhance the NSCs attachment to PHBHHx film in author's lab (data not published). Lipase treatment was even more effective than $\mathrm{NaOH}$ in improving the biocompatibility of PHA and in enhancing the growth of L929 cells on PHA films [30]. Taken together, surface modification is an effective strategy in temporally improvement of hydrophilic property of PHA.

Some other methods have also been recruited to improve the hydrophilic property of PHA, such as electrospinning [36], blending with other material [37] and bio-modification with different proteins [38-40]. Lee's group from Korea fabricated PHBV as nanofibrous mats by electrospinning and chondrocytes derived from rabbit ear were cultured on a PHBV cast film and an electrospun PHBV nano-fibrous mat. Their results showed that on the surface of the electrospun PHBV fabric, more chondrocytes were attached and appeared to have a much greater spreaded morphology than did that of the flat PHBV cast film in the early culture stage. They concluded that electrospun PHBV nanofabric provides an attractive structure for the attachment and growth of chondrocytes as cell culture surfaces [36]. Wang and his colleagues from China showed that gelatin blending increased the surface porosity and decreased the crystallinity of PHBHHx that consequently benefited the cell growth. They also suggested that $10 \%$ gelatin blending had less disruption to PHBHHx spatial structure and resulting in better tensile mechanical properties. All these indicated that gelatin incorporation may improve the performances of PHBHHx to meet the need of different situations during medical implantation [37]. Recently, bio-modification of PHA turned to be a rising star in this field that was very promising to improve the wetting and adhesion property of polymer surface. Several amphiphilic proteins, including PHA repressor protein (PhaR) [38] and PHA granule-associated proteins PhaP [39,40] were applied to develop bio-modified PHA. PhaP and PhaP fusion proteins were able to absorb onto the surface of both natural PHA material and some other artificial hydrophobic materials via hydrophobic interaction. Thus, any functionalized peptide that fused with PhaP could be immobilized on the surface of PHA. Coating with PhaPRGD dramatically increased the surface hydrophilicity of PHBHHx, as well as the attachment of fibroblasts [40]. The attachment, cell proliferations and in vitro differentiation of human bone marrow MSCs were also remarkably enhanced in PhaP-RGD modified PHBHHx scaffold [39].

\section{PHAS MEDIATED NEURAL REGENERATION}

Neural regeneration has received a great deal of attention in both medical and bio-material areas recently. Over the past few decades, many strategies have been attempted to enhance neural regeneration. Unfortunately, none of them has been demonstrated is clinically efficient. Tissue engineering has evolved a new perspective on the current neural regeneration technology [47]. Many possibilities to tailor-made PHA for medical implant applications 
have shown that this class of material has a bright future as tissue engineering material. In addition, the main degradation product of $\mathrm{PHA}, 3-\mathrm{HB}$, has very promising effect in promoting the viability and proliferation of various types of cells, such as osteoblasts, fibroblasts and glial cells, and inhibiting cell apoptosis via triggering the inwards calcium current [48-50]. Our unpublished data also suggested that 3 -HB could promote the survival and neuronal differentiation of NSCs and NPCs. This property made the biodegradable PHA that containing 3-HB become even more attractive for tissue engineering applications. Many members of PHA family, like PHB, PHBHHx, PHBVHHx, have already been attempted for neural regeneration.

\subsection{PHAs Used for Nerve Regeneration in Peripheral Nervous System}

Peripheral nervous system (PNS) injuries are very common in clinical practice and often lead to permanent disability [51-54]. PNS injury results in partial or total loss of motor, sensory and autonomic functions conveyed by the lesioned nerves to the denervated segments of the body, the interruption of axons continuity, degeneration of nerve fibers distal to the lesion and eventual death of axotomized neurons. After peripheral nerve injuries, the capability of severed axons to regenerate and recover functional connections is dependent on several factors, such as the age of the subject, the nerve trunk affected, the site and type of lesion, and the distance over which axons must regrow to span the injury. It is generally considered that in humans, for nerve gaps of less than $2 \mathrm{~cm}$, neurological recovery is moderate, but for gaps longer than $4 \mathrm{~cm}$, recovery is minimal to non-existent [55]. Although surgical methods include allograft, autologous and allogenous organs or tissues including blood vessels and skeletal muscles have been investigated, few successes have been achieved so far.

By applying the principles across the fields of bioengineering, material science and life sciences, tissue engineering appeared to be an effective method for nerve repair. The ideal material for neural regeneration should be biodegradable and must exhibit good biocompatibility with extremely low inflamematory, immunogenic, and cytotoxic responses. In addition, it will be desirable if the conduit presents a porous wall that mimics the size scales of fibers composing the extracellular matrix (ECM) of native tissue and organs, being permeable to the entry of nutrients into the conduit lumen but presenting the necessary barrier to prevent the infiltration of unwanted tissues into the conduit from outside [56].

Compared with the nonbiodegradable and nonpermeable biomaterial tubular silicone prostheses, which has already been used to bridge gaps between severed nerve stumps, PHAs meet almost all the requirements and could be a good candidate to be used as supporting material in nerve regeneration. Some members of PHA have already been applied for repairing of PNS injury (Table 2).

PHB is the first reported member of PHAs. A nonwoven PHB sheet has been used as a wrap to repair transected superficial radial nerves in cats $[57,58]$. The axonal regeneration was demonstrated to be comparable with closure with an epineural suture for a nerve gap of 2 $3 \mathrm{~mm}$ and the inflammatory response was normal. PHB and PHBHHx conduits have also been reported to bridge an irreducible gap up to $10 \mathrm{~mm}$ in rat sciatic nerve where good regeneration and biocompatibility have achieved [59]. The rate and amount of regeneration in the PHB conduit did not fully match the autologous nerve graft but it demonstrated good axonal regeneration with low level of inflammatory infiltration, which is desirable to prevent adhesions to surrounding structures. The difference of the rate and amount of axonal regeneration between PHB conduits group and nerve grafts group was considered to due to the cellular elements inherently present in nerve grafts, such as neurotrophic factors synthesized by de-differentiated Schwann cells. Thus it was speculated that the level of regeneration in a PHB conduit may be further improved by the addition of growth factors. Mohanna et al. developed a novel artificial nerve guide composed of PHB filled with glial growth factor (GGF) and evaluated the long nerve gap repairs effect assisted by GGF containing PHB conduit or empty PHB conduit in a $2-4 \mathrm{~cm}$ rabbit peroneal nerve injury model. The quantity of Schwann cell regeneration was significantly greater in the GGF grafts and the addition of GGF also significantly increased the quantity of axonal regeneration. However, the result of functional recovery is similar to the autograft $[60,61]$.

Recently, PHB was investigated as an alternative to epineural suturing in the treatment of peripheral nerve injuries at the wrist/forearm level of the arm in a randomised, assessor-blinded clinical study [62]. The results demonstrated that PHB can be regarded as a safe alternative for microsurgical epineural suturing since no adverse events or complications considered as product related were found. The sensory recovery and parts of the manual muscle test suggested that treating with PHB may be advantageous as compared to epineural suturing. However, based on the small amount of patients (twelve patients) involved in the study, the results should be confirmed by further large-scale efficacy studies.

PHBHHx was also investigated for possible application in PNS regeneration, which was fabricated to conduits with uniform wall porosity and non-uniform wall porosity and was applied to bridge the $10 \mathrm{~mm}$ defects in the sciatic nerve of Sprague-Dawley (SD) rats [63]. PHBHHx conduits showed good permeability to glucose, 
Table 2. Strategies used for neural regeneration in PNS.

\begin{tabular}{|c|c|c|c|c|}
\hline Material & Neural injury & Strategy & Effect & Ref. \\
\hline \multirow{5}{*}{ PHB } & long peroneal nerve gap & $\begin{array}{l}\text { PHB filled with glia growth factor } \\
\text { (GGF) suspended in alginate hydrogel }\end{array}$ & GGF stimulates a progressive regeneration & [60] \\
\hline & $\begin{array}{l}\text { transected superficial radial } \\
\text { nerves }\end{array}$ & PHB sheets & $\begin{array}{l}\text { comparable with closure with an epineural } \\
\text { suture }\end{array}$ & [57] \\
\hline & $\begin{array}{l}\text { bilaterally sectioned } \\
\text { superficial radial nerves }\end{array}$ & PHB sheets & similar with epineurally suturing of the nerve & [58] \\
\hline & resected in rat sciatic nerve & PHB conduits & comparable with autografts & [59] \\
\hline & $\begin{array}{l}\text { long nerve gaps (up to } 4 \mathrm{~cm} \text { ) } \\
\text { in rabbits' peroneal nerve }\end{array}$ & PHB conduits & $\begin{array}{l}\text { support axonal regeneration across long nerve } \\
\text { gaps }\end{array}$ & [61] \\
\hline PHBHHx & $\begin{array}{l}10 \mathrm{~mm} \text { defects in sciatic } \\
\text { nerve }\end{array}$ & porous PHBHHx conduits & performed similar to the autograft & [63] \\
\hline $\begin{array}{l}\text { PHBHHx } \\
\text { PHBVHHx }\end{array}$ & nerve injury & PHA scaffolds seeded with hBMSC & $\begin{array}{l}\text { promoted cell adnesion proliferation and } \\
\text { differentiation }\end{array}$ & [64] \\
\hline
\end{tabular}

lysozyme and bovine serum albumin, indicating the suitability of the conduits for free exchanges of nutrients. The conduits with non-uniform wall porosity showed better mechanical properties over those of conduits with uniform wall porosity. The $10 \mathrm{~mm}$ gap removed by surgery was found to have been bridged by the regenerated tissue 1 month after the implantation and regenerated myelinated fibers could be observed in both nerve conduits. Nerve fibers grew better in conduits with non-uni form wall porosity, and the regenerated nerves had a similar structure to the autograft controls. The average diameter of the muscle fibers of conduits with non-uniform wall porosity was much larger than that of the conduits with uniform wall porosity, suggesting a faster nerve functional recovery and mitigated atrophy of rat gastrocnemius muscle in the conduits with non-uniform wall porosity. The functional recovery of the conduits with non-uniform wall porosity performed similar to the autograft controls and better than the conduits with uniform wall porosity did. The conduits with uniform wall porosity and the conduits with non-uniform wall porosity lost $24 \%$ and $20 \%$ of their original weight average molecular weights, respectively, indicating a significant in vivo degradation of the polymer conduits.

PHBVHHx scaffolds with different pore size have also been used to repair nerve injury with hBMSC. Efforts have been made to alter the pore sizes. PHBVHHx scaffolds with pore size between $30-60 \mu \mathrm{m}$ might be useful in nerve tissue engineering as it could induce the hBMSC to differentiate into nerve cells and also enhance the cell proliferation in a certain level [64]. Therefore, PHAs have best satisfied the requisites for PNS regeneration due to the better conducting of nerve growth.

\subsection{PHAs Used for Neural Regeneration in the Central Nervous System}

Central nervous system (CNS) injuries, such as traumatic brain injury (TBI), spinal cord injury (SCI) and stroke, can be devastating to the injured person and his/her family. It is estimated that spinal cord injuries alone affect 10,000 each year. Damage to CNS occurs not only in the axons or dendrites but also the cell body. The consequences of injury are not just a break in communication between healthy neurons, but a cascade of events that can lead to neuronal degeneration and cell death. The CNS has little ability to regenerate and repair damaged neurons due to the lack of proliferative Schwann cells. Furthermore, neurons are unable to regenerate correct axonal and dendritic connections. Therefore both the conduit of nerve growth and complementing enough number of neural cells with proper phenotypes should be equally concerned for CNS neural regeneration.

Currently, many kinds of cells, such as Schwann cells [65], olfactory ensheathing cells [66] and bone marrow stromal cells [67], especially the mother cells of nervous system-NSCs, have been transplanted attempting to repair the damage of CNS in the last few decades, and the supportive environment has been modified to produce extracellular matrix molecules, integrins and trophic factors. However, it is still very hard to promote grafted cells survival and proliferation in situ, and further control those cells to differentiate into proper phenotypes. Moreover, in many cases, it is also necessary to bridge grafts by providing a physical substrate for directed axonal growth across the lesion gap and to allow axons to re-enter the host tissue in the appropriate location. Thus, challenges are there and optimized condition or tech- 
niques are needed.

As we described above, tissue engineering has revolutionized the current neural regeneration technology and a subfield of neural tissue engineering is becoming a pioneering interdisciplinary field dedicated to the discovery of new ways to recover nerve functionality after injury. Neural regeneration in CNS is different from that in the PNS. An ideal biomaterial for stimulating CNS regeneration should mimic the mechanical properties, physical dimensions and electrical conductivity of native axons. It must have sufficient biocompatibility for successful brain implantation and must limit glial scar formation so that tissue functionality can be maximized. Several types of materials, such as hydrogel [68,69], collagen [70], Poly (glycerol sebacate) (PGS) [71], Polysialic acid (PSA) [72], and PHAs, have been applied for conducting the nerve growth in CNS neural regeneration. In comparison with other materials, the biodegradable PHAs have shown a superior performance. They not only could offer mechanical support for axon growth, but also have a good interaction with the seeded cells therefore enhance the functional recovery (Table 3).

PHB scaffold combined with Schwann cells has been used to repair the spinal cord injury. During transplantation, adult Schwann cells suspension was added to the PHB, 4 weeks after, PHB conduit was well-integrated into posttraumatic cavity, and the neurofilament-positive axons filled the conduit and became associated with the implanted cells [73]. PHB fibers coated with alginate hydrogel and fibronectin was used as carrier scaffold for matrix components. After implanted into the lesion cavity, cell loss significantly reduced and a rescuing effect similar to the treatment with BDNF or NT-3 was obtained. Combining PHB fibers with neonatal Schwann cells, the therapeutic effect was even better as the regenerating axons were seen to enter the graft from both ends and to extend along its entire length [74]. PHBV microspheres containing alginate hydrogel served as 3-dimensional cell carrier for various types of nerve cells has been proved are suitable scaffolds for neural tissue engineering. They supported the adhesion, growth, proliferation and differentiation of neuro2a neuroblastoma cells and fetal cortical neurons. In addition, the primary neurons were found to extend neurites on PHBV microspheres, indicating that PHBV enhanced the neuronal maturation and promoted greater axon-dendrite segregation [75]. An emerging star in PHAs family, PHBHHx, has also been demonstrated to have superior compatibility with NSCs and NPCs. PHBHHx nanofiber scaffolds, structurally similar to natural ECM, supported the attachment and survival of NSCs and NPCs. In comparison with other members of PHAs like PHB and PHB4HB, three dimensional PHBHHx nanofiber matrices appeared to be more suitable in promoting neuronal differentiation and synaptogenesis of NSCs and NPCs in vitro. It therefore could be a very promising biomaterial for CNS regeneration [27]. Our unpublished data also shown that the stemness of NSCs and NPCs, in terms of proliferation and multiple differentiations, were well maintained on alkali treated PHBHHx films in a modified culture system. This makes it possible to obtain enough number of transplantable progenitor cells that are essential for functional recovery. In addition, our in vivo observation shown that there is no severe glial scar formed after implanting the PHBHHx-NSCs/NPCs complex into TBI rat brain. It suggested that implanting PHBHHx-NSCs/NPCs complex might be able to promote the neurites extension and functional recovery. Combined all these data together, it points that, in comparison with other materials, PHAs might be a better choice for CNS regeneration due to its higher bio-compatibility with NSCs.

\section{CONCLUSIONS AND FUTURE PERSPECTIVE OF PHAs IN NEURAL REGENERATION}

Up to now, the functional recovery after neural injury is still hindered by a lack of knowledge of target cells dif-

Table 3. Members of PHAs used for neural regeneration in CNS.

\begin{tabular}{|c|c|c|c|c|}
\hline Material & Neural injury & Strategy & Effect & Ref \\
\hline PHB & cervical spinal cord injury & PHB scaffolf seeded with Schwann cells & $\begin{array}{l}\text { increased cell proliferation } \\
\text { and axonal regeneration }\end{array}$ & {$[73]$} \\
\hline PHB & spinal cord injury & $\begin{array}{c}\text { PHB scalffold containing alginate hydrogel, } \\
\text { fibronectin served as a carrier for Schwann } \\
\text { cells }\end{array}$ & $\begin{array}{l}\text { enhanced the neuronal } \\
\text { survival and regeneration }\end{array}$ & {$[74]$} \\
\hline PHBV & $\begin{array}{l}\text { neurodegenerative diseases \& } \\
\text { traumatic injuries }\end{array}$ & $\begin{array}{l}\text { PHBV microsphere was used as suppoting } \\
\text { scaffold }\end{array}$ & $\begin{array}{c}\text { supported PC12, NPCs } \\
\text { neurons and promoted } \\
\text { greateraxon-dendrite } \\
\text { segregation }\end{array}$ & {$[75]$} \\
\hline PHB \& P3HB4HB & central nervous system injury & $\begin{array}{l}\text { PHA nanofiber scaffolds seeded with neural } \\
\text { stem cells (NSCs) }\end{array}$ & $\begin{array}{l}\text { promoted NSC growth and } \\
\text { differentiation }\end{array}$ & {$[27]$} \\
\hline РНBHHx \& PHA & pathological injury & rCGNs seeded on electrospun PHA film & promoted cell viability & {$[76]$} \\
\hline
\end{tabular}


ferentiation and neural network construction. With combined efforts of scientists and engineers from a variety of disciplines, experimental work in this field has made a great progress. Several successful studies both in vitro and in vivo have clearly demonstrated that neural tissue engineering displayed a very promising blueprint for neural regeneration. Among the several kinds of biomaterials, the biodegradable and biocompatible materials, like PHAs, might be an ideal candidate that could be used to induce target cell differentiation, conduit nerve extension as well as offer mechanical support. The existed data had shown that some members of PHAs facilitated neural regeneration after a certain modification. However, no single material is enough to improve the properties for neural regeneration yet. Same materials in different spatial structure also have different influence on the behavior of seeded cells. Hence, the fields of both approaches for improving the properties of PHA and the strategies for neural regeneration are still wide open to get the most appropriate way for effective neural regeneration in vivo. Combining NSCs and NPCs with the neurotrophic factors coated $\mathrm{PHBHHx}$ films to treat ischemic brain damage are currently ongoing in author's laboratory. Our preliminary data has shown that a greater number of NSCs and NPCs survived and preferably differentiated into neurons when cultured on top of coated films. Further observation in regarding the effect in vivo and the mechanisms is required. In summary, surface/ structure modified PHAs combined with bio-functionalization should be a promising strategy to develop the novel scaffold for neural regeneration/nerve tissue engineering.

\section{ACKNOWLEDGEMENTS}

This work has been supported by grants from the National Natural Science Foundation of China $(81070998,31070943,81172170)$ and the Fundamental Research Funds for the Central Universities from Xi'an Jiaotong University.

\section{REFERENCES}

[1] Johansson, C.B., Momma, S., Clarke, D.L., Risling, M., Lendahl, U. and Frisén, J. (1999) Identification of a neural stem cell in the adult mammalian central nervous system. Cell, 96, 25-34. doi:10.1016/S0092-8674(00)80956-3

[2] Widenfalk, J., Lundstromer, K., Jubran, M., Brene, S. and Olson, L. (2001) Neurotrophic factors and receptors in the immature and adult spinal cord after mechanical injury or kainic acid. Journal of Neuroscience, 21, 34573475 .

[3] Cai, D., Shen, Y., De Bellard, M., Tang, S. and Filbin, M.T. (1999) Prior exposure to neurotrophins blocks inhibition of axonal regeneration by MAG and myelin via a cAMP-dependent mechanism. Neuron, 22, 89-101.
doi:10.1016/S0896-6273(00)80681-9

[4] Miya, D., Giszter, S., Mori, F., Adipudi, V., Tessler, A. and Murray, M. (1997) Fetal transplants after the development of function after spinal cord transection in newborn rats. Journal of Neuroscience, 17, 4856-4872.

[5] Diener, P.S. and Bregman, B.S. (1998) Fetal spinal cord transplants support growth of supraspinal and segmental projections after cervical spinal cord hemisection in the neonatal rat. Journal of Neuroscience, 18, 779-793.

[6] Tsuji, O., Miura, K., Fujiyoshi, K., Momoshima, S., Nakamura, M. and Okano, H. (2011) Cell therapy for spinal cord injury by neural stem/progenitor cells derived from iPS/ES cells. Neurotherapeutics, 8, 668-676. doi:10.1007/s13311-011-0063-z

[7] Arias-Carrion, O. and Yuan, T.F. (2009) Autologous neural stem cell transplantation: A new treatment option for Parkinson's disease? Med Hypotheses, 73, 757-759. doi:10.1016/j.mehy.2009.04.029

[8] Lepore, A.C. and Fischer, I. (2005) Lineage-restricted neural precursors survive, migrate, and differentiate following transplantation into the injured adult spinal cord. Experimental Neurology, 194, 230-242. doi:10.1016/j.expneurol.2005.02.020

[9] Lepore, A.C., Han, S.S., Tyler-Polsz, C.J., Cai, J., Rao, M.S. and Fischer, I. (2004) Differential fate of multipotent and lineage-restricted neural precursors following transplantation into the adult CNS. Neuron Glia Biology, 1, 113-126. doi:10.1017/S1740925X04000213

[10] Lepore, A.C., Neuhuber, B., Connors, T.M., Han, S.S., Liu, Y., Daniels, M.P., Rao, M.S. and Fischer, I. (2006) Long-term fate of neural precursor cells following transplantation into developing and adult CNS. Neuroscience, 142, 287-304. doi:10.1016/j.neuroscience.2005.12.067

[11] Thonhoff, J.R., Lou, D.I., Jordan, P.M., Zhao, X. and Wu, P. (2008) Compatibility of human fetal neural stem cells with hydrogel biomaterials in vitro. Brain Research, 1187, 42-51. doi:10.1016/j.brainres.2007.10.046

[12] Xiong, Y., Zeng, Y.S., Zeng, C.G., Dum, B.L., He, L.M., Quan, D.P., Zhang, W., Wang, J.M., Wu, J.L., Li, Y. and Li, J. (2009) Synaptic transmission of neural stem cells seeded in 3-dimensional PLGA scaffolds. Biomaterials, 30, 3711-3722. doi:10.1016/j.biomaterials.2009.03.046

[13] Park, K.I., Teng, Y.D. and Snyder, E.Y. (2002) The injured brain interacts reciprocally with neural stem cells supported by scaffolds to reconstitute lost tissue. Nature Biotechnology, 20, 1111-1117. doi:10.1038/nbt751

[14] Chen, G.Q. and Q. Wu, (2005) The application of polyhydroxyalkanoates as tissue engineering materials. Biomaterials, 26, 6565-6578. doi:10.1016/j.biomaterials.2005.04.036

[15] Steinbuchel, A. and Valentin, H.E. (1995) Diversity of bacterial polyhydroxyalkanoic acids. FEMS Microbiology Letters, 128, 219-228. doi:10.1111/j.1574-6968.1995.tb07528.x

[16] Wu, T.P. Hu, P., Zhang, X.B., Li, W. and Chen, F. (2004) Biocompatibility of modified poly-beta-hydroxybutyric acid to adrenocortical cells. Journal of Wuhan University of Technology-Materials Science Edition, 19, 38-40. doi:10.1007/BF03000164 
[17] Nebe, B., Forster, C., Pommerenke, H., Fulda, G., Behrend, D., Bernewski, U., Schmitz, K.P. and Rychly, J. (2001) Structural alterations of adhesion mediating components in cells cultured on poly bhydroxybutyric acid. Biomaterials, 22, 2425-2434. doi:10.1016/S0142-9612(00)00430-0

[18] Shishatskaya, E.I. and Volova, T.G. (2004) A comparative investigation of biodegradable polyhydroxyalkanoate films as matrices for in vitro cell cultures. Journal of Materials Science: Materials in Medicine, 15, 915-923. doi:10.1023/B:JMSM.0000036280.98763.c1

[19] Kuppan, P., Vasanthan, K.S., Sundaramurthi, D., Krishnan, U.M. and Sethuraman, S. (2011) Development of poly(3-hydroxybutyrate-co-3-hydroxyvalerate) fibers for skin tissue engineering: Effects of topography, mechanical, and chemical stimuli. Biomacromolecules, 12, 31563165. doi: $10.1021 / \mathrm{bm} 200618 \mathrm{w}$

[20] Kenar, H., Kocabas, A., Aydinli, A. and Hasirci, V. (2008) Chemical and topographical modification of PHBV surface to promote osteoblast alignment and confinement. Journal of Biomedical Materials Research: Part A, 85, 1001-1010. doi:10.1002/jbm.a.31638

[21] Doi, Y., Kitamura, S. and Abe, H. (1995) Microbial synthesis and characterization of poly(3-hydroxybutyrate-co3-hydroxyhexanoate). Macromolecules, 28, 4822-4828. doi: $10.1021 / \mathrm{ma} 00118 \mathrm{a} 007$

[22] Qu, X.H., Wu, Q., Liang, J., Zou, B. and Chen, G.Q. (2006) Effect of 3-hydroxyhexanoate content in poly(3hydroxybutyrate-co-3-hydroxyhexanoate) on in vitro growth and differentiation of smooth muscle cells. Biomaterials, 27, 2944-2950. doi:10.1016/j.biomaterials.2006.01.013

[23] Wang, Y.W., Yang, F., Wu, Q., Cheng, Y.C., Yu, P.H., Chen, J. and Chen, G.Q. (2005) Effect of composition of poly(3-hydroxybutyrate-co-3-hydroxyhexanoate) on growth of fibroblast and osteoblast. Biomaterials, 26, 755-761. doi:10.1016/j.biomaterials.2004.03.023

[24] Wang, Y., Bian, Y.Z., Wu, Q. and Chen, G.Q. (2008) Evaluation of three-dimensional scaffolds prepared from poly (3-hydroxybutyrate-co-3-hydroxyhexanoate) for growth of allogeneic chondrocytes for cartilage repair in rabbits. Biomaterials, 29, 2858-2868.

doi:10.1016/j.biomaterials.2008.03.021

[25] Wang, Y.W., Wu, Q. and Chen, G.Q. (2004) Attachment, proliferation and differentiation of osteoblasts on random biopolyesterpoly(3-hydroxybutyrate-co-3-hydroxyhexanoate) scaffolds. Biomaterials, 25, 669-675. doi:10.1016/S0142-9612(03)00561-1

[26] Yang, M., Zhu, S., Chen, Y., Chang, Z., Chen, G., Gong, Y., Zhao, N. and Zhang, X. (2004) Studies on bone marrow stromal cells affinity of poly (3-hydroxybutyrate-co3-hydroxyhexanoate). Biomaterials, 25, 1365-1373. doi:10.1016/j.biomaterials.2003.08.018

[27] Xu, X.Y., Li, X.T., Peng, S.W., Xiao, J.F., Liu, C., Fang, G., Chen, K.C. and Chen, G.Q. (2010) The behaviour of neural stem cells on polyhydroxyalkanoate nanofiber scaffolds. Biomaterials, 31, 3967-3975. doi:10.1016/j.biomaterials.2010.01.132

[28] Liang, Y.S., Zhao, W. and Chen, G.Q. (2008) Study on the biocompatibility of novel terpolyester poly (3-hydroxybtyrate-co-3-hydroxyvalerate-co-3-hydroxy-hexanoate).
Journal of Biomedical Materials Research: Part A, 87, 441-449. doi:10.1002/jbm.a.31801

[29] Hu, Y.J., Wei, X., Zhao, W., Liu, Y.S. and Chen, G.Q. (2009) Biocompatibility of poly(3-hydroxybutyrate-co-3hydroxyvalerate-co-3-hydroxyhexanoate) with bone marrow mesenchymal stem cells. Acta Biomaterialia, 5, 1115-1125. doi:10.1016/j.actbio.2008.09.021

[30] Yang, X., Zhao, K. and Chen, G.Q. (2002) Effect of surface treatment on the biocompatibility of microbial polyhydroxyalkanoates. Biomaterials, 23, 1391-1397. doi:10.1016/S0142-9612(01)00260-5

[31] Shen, F., Zhang, E. and Wei, Z. (2010) In vitro blood compatibility of poly (hydroxybutyrate-co-hydroxyhexanoate) and the influence of surface modification by alkali treatment. Materials Science and Engineering C, 30, 369375. doi:10.1016/i.msec.2009.12.003

[32] Ma, Z., Gao, C., Gong, Y. and Shen, J. (2003) Chondrocyte behaviors on poly-L-lactic acid (PLLA) membranes containing hydroxyl, amide or carboxyl groups. Biomaterials, 24, 3725-3730. doi:10.1016/S0142-9612(03)00247-3

[33] Shangguan, Y.Y., Wang, Y.W., Wu, Q. and Chen, G.Q. (2006) The mechanical properties and in vitro biodegradation and biocompatibility of UV-treated poly(3-hydroxybutyrate-co-3-hydroxyhexanoate). Biomaterials, 27, 23492357. doi:10.1016/j.biomaterials.2005.11.024

[34] Ren, Y., Wang, C. and Qiu, Y. (2008) Aging of surface properties of ultra high modulus polyethylene fibers treated with $\mathrm{He} / \mathrm{O}_{2}$ atmospheric pressure plasma jet. Surface and Coatings Technology, 202, 2670-2676. doi:10.1016/j.surfcoat.2007.09.043

[35] Benavente, J. and Vazquez, M.I. (2004) Effect of age and chemical treatments on characteristic parameters for active and porous sublayers of polymeric composite membranes. Journal of Colloid and Interface Science, 273, 547-555. doi:10.1016/j.jcis.2003.11.023

[36] Lee, I.S., Kwon, O.H., Meng, W., Kang, I.K. and Ito, Y. (2004) Nanofabrication of microbial polyester by electrospinning promotes cell attachment. Macromolecular Research, 12, 374-378. doi:10.1007/BF03218414

[37] Wang, Y.W., Wu, Q. and Chen, G.Q. (2005) Gelatin blending improves the performance of poly(3-hydroxybutyrate-co-3-hydroxyhexanoate) films for biomedical application. Biomacromolecules, 6, 566-571. doi: $10.1021 / \mathrm{bm} 049342 \mathrm{~d}$

[38] Dong, C.L., Li, S.Y., Wang, Y., Dong, Y., Tang, J.Z., Chen, J.C. and Chen, G.Q. (2012) The cytocompatability of polyhydroxyalkanoates coated with a fusion protein of PHA repressor protein (PhaR) and Lys-Gln-Ala-GlyAsp-Val (KQAGDV) polypeptide. Biomaterials, 33, 2593-2599. doi:10.1016/j.biomaterials.2011.12.020

[39] You, M., Peng, G., Li, J., Ma, P., Wang, Z., Shu, W., Peng, S. and Chen, G.Q. (2011) Chondrogenic differenttiation of human bone marrow mesenchymal stem cells on polyhydroxyalkanoate (PHA) scaffolds coated with PHA granule binding protein PhaP fused with RGD peptide. Biomaterials, 32, 2305-2313. doi:10.1016/j.biomaterials.2010.12.009

[40] Dong, Y., Li, P., Chen, C.B., Wang, Z.H., Ma, P. and Chen, G.Q. (2010) The improvement of fibroblast growth 
on hydrophobic biopolyesters by coating with polyhydroxyalkanoate granule binding protein PhaP fused with cell adhesion motif RGD. Biomaterials, 31, 8921-8930. doi:10.1016/j.biomaterials.2010.08.001

[41] Zhao, K., Deng, Y. and Chen, G.Q. (2003) Effects of surface morphology on the biocompatibility of polyhy-droxyalkanoates. Biochemical Engineering Journal, 16, 115123. doi:10.1016/S1369-703X(03)00029-9

[42] Zheng, Z., Bei, F.F., Tian, H.L. and Chen, G.Q. (2005) Effects of crystallization of polyhydroxyalkanoate blend on surface physicochemical properties and interactions with rabbit articular cartilage chondrocytes. Biomaterials, 26, 3537-3548. doi:10.1016/j.biomaterials.2004.09.041

[43] Li, J., Yun, H., Gong, Y., Zhao, N. and Zhang, X. (2005) Effects of surface modification of poly (3-hydroxy-butyrate-co-3-hydroxyhexanoate) (PHBHHx) on physicochemical properties and on interactions with MC3T3-E1 cells. Journal of Biomedical Materials Research, 75, 985-998. doi:10.1002/jbm.a.30504

[44] Wang, Y.W., Wu, Q. and Chen, G.Q. (2003) Reduced mouse fibroblast cell growth by increased hydrophilicity of microbial polyhydroxyalkanoates via hyaluronan coating. Biomaterials, 24, 4621-4629. doi:10.1016/S0142-9612(03)00356-9

[45] Qu, X.H., Wu, Q., Liang, J., Qu, X., Wang, S.G. and Chen, G.Q. (2005) Enhanced vascular-related cellular affinity on surface modified copolyesters of 3-hydroxy-butyrate and 3-hydroxyhexanoate (PHBHHx). Biomaterials, 26, 6991-7001. doi:10.1016/j.biomaterials.2005.05.034

[46] Li, X.T., Sun, J., Chen, S. and Chen, G.Q. (2008) In Vitro investigation of maleated poly(3-hydroxybutyrate-co-3hydroxyhexanoate) for its biocompatibility to mouse fibroblast L929 and human microvascular endothelial cells. Journal of Biomedical Materials Research. Part A, 87, 832-842. doi:10.1002/jbm.a.31890

[47] Huang, Y.C. and Huang, Y.Y. (2006) Biomaterials and strategies for nerve regeneration. Artifical Organs, 30, 514-522. doi:10.1111/j.1525-1594.2006.00253.x

[48] Zhao, Y., Zou, B., Shi, Z., Wu, Q. and Chen, G.Q. (2007) The effect of 3-hydroxybutyrate on the in Vitro differenttiation of murine osteoblast MC3T3-E1 and in Vivo bone formation in ovariectomized rats. Biomaterials, 28, 30633073. doi:10.1016/j.biomaterials.2007.03.003

[49] Cheng, S., Wu, Q., Zhao, Y., Zou, B. and Chen, G.Q. (2006) Effect of poly(hydroxybutyrate-co-hydroxyhexanoate) microparticles on growth of murine fibroblast L929 cells. Polymer Degradation and Stability, 91, 3191-3196. doi:10.1016/j.polymdegradstab.2006.07.010

[50] Xiao, X.Q., Zhao, Y. and Chen, G.Q. (2007) The effect of 3-hydroxybutyrate and its derivatives on the growth of glial cells. Biomaterials, 28, 3608-3616. doi:10.1016/j.biomaterials.2007.04.046

[51] Ignatiadis, I.A., Yiannakopoulos, C.K., Barbitsioti, A.D., Avram, A.M., Patralexis, H.G., Tsolakis, C.K., Papalois, A.E., Xenakis, T.H., Beris, A.E. and Soucacos, P.N. (2007) Diverse types of epineural conduits for bridging short nerve defects. An experimental study in the rabbit. Microsurgery, 27, 98-104. doi:10.1002/micr.20313

[52] Wang, X., Hu, W., Cao, Y., Yao, J., Wu, J. and Gu, X. (2005) Dog sciatic nerve regeneration across a 30-mm defect bridged by a chitosan/PGA artificial nerve graft. Brain, 128, 1897-1910. doi:10.1093/brain/awh517

[53] Nakayama, K., Takakuda, K., Koyama, Y., Itoh, S., Wang, W., Mukai, T. and Shirahama, N. (2007) Enhancement of peripheral nerve regeneration using bioabsorbable polymer tubes packed with fibrin gel. Artificial Organs, 31, 500-508.

[54] Schnell, E., Klinkhammer, K., Balzer, S., Brook, G., Klee, D., Dalton, P. and Mey J. (2007) Guidance of glial cell migration and axonal growth on electrospun nanofibers of poly-epsilon-caprolactone and a collagen/poly-epsi-loncaprolactone blend. Biomaterials, 28, 3012-3025. doi:10.1016/j.biomaterials.2007.03.009

[55] Reyes, O., Sosa, I., Kuffler D.P. (2005) Promoting neurological recovery following a traumatic peripheral nerve injury. Puerto Rico Health Sciences Journal, 24, $215-$ 223.

[56] Tavangarianan, F. and Li, Y. (2012) Carbon nanostructures as nerve scaffolds for repairing large gaps in severed nerves. Ceramics International, 38, 6075-6090. doi:10.1016/i.ceramint.2012.05.038

[57] Hazari, A., Johansson-Ruden, G., Junemo-Bostrom, K., Ljungberg, C., Terenghi, G., Green, C. and Wiberg, M. (1999) A new resorbable wrap-around implant as an alternative nerve repair technique. Journal of Hand Surgery (European Volume), 24, 291-295. doi:10.1054/jhsb.1998.0001

[58] Ljungberg, C., Johansson-Ruden, G., Bostrom, K.J., Novikov, L. and Wiberg, M. (1999) Neuronal survival using a resorbable synthetic conduit as an alternative to primary nerve repair. Microsurgery, 19, 259-264. doi:10.1002/(SICI)1098-2752(1999)19:6<259::AID-MIC $\underline{\mathrm{R} 1>3.0 . \mathrm{CO} ; 2-\mathrm{Q}}$

[59] Hazari, A., Wiberg, M., Johansson-Ruden, G., Green, C. and Terenghi, G. (1999) A resorbable nerve conduit as an alternative to nerve autograft in nerve gap repair. British Journal of Plastic Surgery, 52, 653-657. doi:10.1054/bjps.1999.3184

[60] Mohanna, P.N., Young, R.C., Wiberg, M. and Terenghi, G. (2003) A composite poly-hydroxybutyrate-glial growth factor conduit for long nerve gap repairs. Journal of Anatomy, 203, 553-565. doi:10.1046/j.1469-7580.2003.00243.x

[61] Young, R.C., Wiberg, M. and Terenghi, G. (2002) Poly3-hydroxybutyrate (PHB): A resorbable conduit for longgap repair in peripheral nerves. British Journal of Plastic Surgery, 55, 235-240. doi:10.1054/bjps.2002.3798

[62] Aberg, M., Ljungberg, C., Edin, E., Millqvist, H., Nordh, E., Theorin, A., Terenghi, G. and Wiberg, M. (2009) Clinical evaluation of a resorbable wrap-around implant as an alternative to nerve repair: A prospective, assessor-blinded, randomised clinical study of sensory, motor and functional recovery after peripheral nerve repair. Journal of Plastic, Reconstructive \& Aesthetic Surgery, 62, 1503 1509. doi:10.1016/j.bjps.2008.06.041

[63] Bian, Y.Z., Wang, Y., Aibaidoula, G., Chen, G.Q. and Wu. Q. (2009) Evaluation of poly(3-hydroxybutyrateco-3-hydroxyhexanoate) conduits for peripheral nerve 
regeneration. Biomaterials, 30, 217-225. doi:10.1016/j.biomaterials.2008.09.036

[64] Wang, L., Wang, Z.H., Shen, C.Y., You, M.L., Xiao, J.F. and Chen, G.Q. (2010) Differentiation of human bone marrow mesenchymal stem cells grown in terpolyesters of 3-hydroxyalkanoates scaffolds into nerve cells. Biomaterials, 31, 1691-1698. doi:10.1016/j.biomaterials.2009.11.053

[65] Oudega, M. and Xu, X.M. (2006) Schwann cell transplantation for repair of the adult spinal cord. Journal of Neurotrauma, 23, 453-467. doi:10.1089/neu.2006.23.453

[66] Raisman, G. and Li, Y. (2007) Repair of neural pathways by olfactory ensheathing cells. Nature Reviews Neuroscience, 8, 312-319.

[67] Enzmann, G.U., Benton, R.L., Talbott, J.F., Cao, Q. and Whittemore, S.R. (2006) Functional considerations of stem cell transplantation therapy for spinal cord repair. Journal of Neurotrauma, 23, 479-495. doi:10.1089/neu.2006.23.479

[68] Crompton, K.E., Goud, J.D., Bellamkonda, R.V., Gengenbach, T.R., Finkelstein, D.I., Horne, M.K. and Forsythe, J.S. (2007) Polylysine-functionalised thermo-responsive chitosan hydrogel for neural tissue engineering. Biomaterials, 28, 441-449. doi:10.1016/j.biomaterials.2006.08.044

[69] Ma, W., Fitzgerald, W., Liu, Q.Y., O'Shaughnessy, T.J., Maric, D., Lin, H.J., Alkon, D.L. and Barker, J.L. (2004) CNS stem and progenitor cell differentiation into functional neuronal circuits in three-dimensional collagen gels. Experimental Neurology, 190, 276-288. doi:10.1016/j.expneurol.2003.10.016

[70] Stang, F., Fansa, H., Wolf, G. and Keilhoff, G. (2005)
Collagen nerve conduits-Assessment of biocompatibility and axonal regeneration. Bio-Medical Materials and Engineering, 15, 3-12.

[71] Sundback, C.A., Shyu, J.Y., Wang, Y., Faquin, W.C., Langer, R.S., Vacanti, J.P. and Hadlock, T.A. (2005) Biocompatibility analysis of poly(glycerol sebacate) as a nerve guide material. Biomaterials, 26, 5454-5464. doi:10.1016/j.biomaterials.2005.02.004

[72] Haile, Y., Haastert, K., Cesnulevicius, K., Stummeyer, K., Timmer, M., Berski, S., Dräger, G., Gerardy-Schahn, R. and Grothe, C. (2007) Culturing of glial and neuronal cells on polysialic acid. Biomaterials, 28, 1163-1173. doi:10.1016/j.biomaterials.2006.10.030

[73] Novikova, L.N., Pettersson, J., Brohlin, M., Wiberg, M. and Novikov, L.N. (2008) Biodegradable poly-beta-hydroxybutyrate scaffold seeded with Schwann cells to promote spinal cord repair. Biomaterials, 29, 1198-1206. doi:10.1016/j.biomaterials.2007.11.033

[74] Novikov, L.N., Novikova, L.N., Mosahebi, A., Wiberg, M., Terenghi, G. and Kellerth, J.O. (2002) A novel biodegradable implant for neuronal rescue and regeneration after spinal cord injury. Biomaterials, 23, 3369-3376. doi:10.1016/S0142-9612(02)00037-6

[75] Chen, W. and Tong, Y.W. (2012) PHBV microspheres as neural tissue engineering scaffold support neuronal cell growth and axon-dendrite polarization. Acta Biomaterialia, 8, 540-548. doi:10.1016/j.actbio.2011.09.026

[76] Yu, B.Y., Chen, C.R., Sun, Y.M., Young, T.H. (2009) The response of rat cerebellar granule neurons (rCGNs) to various polyhydroxyalkanoate (PHA) films. Desalination, 245, 639-646. doi:10.1016/j.desal.2009.02.031. 